\title{
ARTIGOS
}

Submetido 01.11.2014. Aprovado 12.05.2015

Avaliado pelo processo de double blind review. Este artigo tem coautoria de membro do Corpo Editorial da RAE, foi avaliado em double blind review, com isenção e independência. Editores Científicos: Delane Botelho e Edgard Barki DOI: http://dx.doi.org/10.1590/So034-759020150609

\section{PERCEPÇÃO DA PRESENÇA DOS OUTROS CONSUMIDORES E SUA RELAÇÃO COM EMOÇÕES E VALOR HEDÔNICO DE COMPRA}

\author{
Perception of other customers' presence and its relationship with emotions and \\ hedonic shopping value
}
Percepción de la presencia de los otros consumidores y su relación con emociones y valor hedónico de compra

\section{RESUMO}

Este artigo investiga a relação da percepção da presença de outros clientes com as emoções e o valor hedônico, resultantes da compra em ambiente de varejo popular. Por meio de um levantamento com 247 clientes e uso de modelagem de equações estruturais, os resultados indicam um efeito significativo da presença de outros consumidores sobre as respostas individuais do consumidor durante o processo de compra. 0 estudo traz uma contribuição importante para a área acadêmica do comportamento do consumidor em ambientes de varejo, uma vez que a validação da escala Other Customers Perception (OCP) no varejo tradicional amplia as possibilidades de uma melhor compreensão do impacto da dimensão social do ambiente varejista em uma variedade de contextos. Ele também apoia o argumento de que a experiência de consumo envolve mais do que uma simples avaliação do desempenho do varejista, chamando a atenção para os fatores sociais constitutivos do ambiente de varejo.

PALAVRAS-CHAVE | Percepção dos outros consumidores, varejo popular, emoções, valor hedônico de compra, ambiente de varejo.

\begin{abstract}
This paper investigates how the perception of other customers' presence relates to emotions and hedonic shopping value in a popular retail environment. We conducted a survey with 247 customers and used structural equation modeling, and found a significant effect of other customers' presence on individual consumer response in the purchasing process. This study makes an important contribution to the field of consumer behavior in retail environments, since the validation of the scale (Other Customers' Perception) in traditional retail allows the possibility to better understand the impact of retail environment's social dimension in a variety of contexts. It also corroborates that consumer experience involves more than simply assessing retailers' performance, drawing attention to social factors in the retailing environment.
\end{abstract}

KEYWORDS I Other customers' presence, popular retailing, emotions, hedonic shopping value, retail environment.

\section{EDVAN CRUZ AGUIAR}

edvan.ed@gmail.com

Doutorando em Administração de

Empresas pela Universidade Federal de Pernambuco, Programa de Pós-Graduação em Administração Recife - Pernambuco - PE, Brasil

\section{SALOMÃO ALENCAR DE FARIAS} saf@ufpe.br Professor da Universidade Federal de Pernambuco, Programa de Pós-Graduação em Administração Recife - Pernambuco - PE, Brasil

\section{RESUMEN}

Este artículo investiga la relación de la percepción de la presencia de otros clientes con las emociones y el valor hedónico, resultantes de la compra en ambiente de comercio popular. Por medio de un relevamiento con 247 clientes y uso de modelado de ecuaciones estructurales, los resultados indican un efecto significativo de la presencia de otros consumidores sobre las respuestas individuales del consumidor durante el proceso de compra. El estudio resulta en una contribución importante para el área académica sobre el comportamiento del consumidor en ambientes de comercio, ya que la validación de la escala Other Customers Perception (OCP) en el comercio tradicional amplía las posibilidades de una mejor comprensión del impacto de la dimensión social del ambiente minorista en una variedad de contextos. Él también apoya el argumento de que la experiencia de consumo involucra más que una simple evaluación del desempeño del comerciante, llamando la atención hacia los factores sociales constitutivos del ambiente de comercio.

PALABRAS CLAVE I Percepción de los otros consumidores, comercio popular, emociones, valor hedónico de compra, ambiente de comercio. 


\section{INTRODUÇÃO}

Em boa parte das relações de troca no varejo tradicional, clientes compartilham o mesmo espaço onde produtos e serviços são ofertados e/ou consumidos. Percebe-se que, para além dos fatores sensoriais constitutivos da atmosfera de loja, a presença de outras pessoas pode interferir nas respostas individuais do consumidor, sejam elas de caráter atitudinal, afetivo ou comportamental (Aguiar \& Gomes, 2014; Baker, 1986; Brandão \& Parente, 2012; Eroglu, Machleit, \& Barr, 2005; Kim \& Kim, 2012; Turley \& Milliman, 2000).

A dimensão social do ambiente varejista tem sido considerada capaz de influenciar atitudes e comportamentos, tanto de clientes como do pessoal de linha de frente (Baker, 1986; Bitner, 1990; 1992; Turley \& Milliman, 2000). Ela pode interferir na comunicação da imagem da empresa e nos interesses de seus clientes. Assim, entender o comportamento do consumidor em ambientes varejistas também envolve examinar as influências sociais (Yüksel, 2009). Tem-se observado, na literatura, pesquisas que objetivam melhor entender como os clientes influenciam uns aos outros (exs.: Grove \& Fisk, 1997; Martin, 1996; McGrath \& Otnes, 1995; Tombs \& McColl-Kennedy, 2003, 2010, 2013).

A teoria do impacto social (SIT [Social Impact Theory]) caracteriza as muitas maneiras como indivíduo(s) afeta $(\mathrm{m})$ outro(s) em função de aspectos como a restrição de espaço físico e, especificamente, como o impacto sobre as pessoas é moderado pela importância, pela proximidade e pelo número de outros indivíduos em um dado ambiente (Latané, 1981). 0 impacto social, definido como qualquer influência sobre os sentimentos, pensamentos e comportamentos individuais que é exercida por uma presença real, insinuada ou imaginada, ou por ações de outros indivíduos, corresponde a um aspecto relevante a ser investigado e ainda pouco explorado em contextos varejistas (Argo, Dahl, \& Manchanda, 2005).

Apreende-se que a percepção da presença de "outros consumidores", que são geralmente desconhecidos, tem o potencial de melhorar ou diminuir avaliações individuais acerca da experiência de consumo e do varejista. Brocato, Voorhees, e Baker (2012) desenvolveram uma escala que mensura o construto denominado Other Customer Perception (OCP [Percepção dos Outros Consumidores]), a qual se propõe a avaliar as percepções que o indivíduo tem dos outros consumidores durante experiências de consumo.

Os "outros consumidores" referem-se a clientes que estão simultaneamente no mesmo ambiente de varejo com o consumidor que percebe sua presença. Eles têm sido considerados capazes de contribuir para que experiências estéticas no ambiente varejista sejam estabelecidas (Nuttavuthisit, 2014), e a argumentação teórica proposta por Latané (1981) mostra-se útil para o entendimento da maneira como outros clientes podem influenciar atitudes e comportamentos individuais.
Quando se estuda a relação entre ambiente de consumo e comportamento do consumidor, geralmente a atenção volta-se para os fatores sensoriais e estéticos como determinantes das respostas individuais do cliente, sendo ainda relativamente poucos os estudos que investigam o papel dos fatores sociais. Argumenta-se que a experiência de compra também é impactada pela maneira como pistas sociais do ambiente são interpretadas (Breazeale \& Ponder, 2013).

Considerando a presença social aspecto importante no estudo da relação entre atmosfera de loja e comportamento do consumidor, somando-se a isso a identificação de apenas um estudo no contexto brasileiro que pesquisou o construto OCP (Araujo, Grillo, \& Espartel, 2013), o presente trabalho propôs-se a investigar a relação da percepção da presença de outros clientes com as emoções e o valor hedônico, resultantes da compra em ambiente de varejo popular.

Ressalta-se, ainda, que a maioria dos estudos que investigam o ambiente social geralmente utiliza contextos de serviços. Espera-se que a aplicação e validação desse instrumento em ambiente de varejo tradicional traga uma contribuição importante para a área de estudo, pois amplia as possibilidades de investigar o impacto da presença social nos mais variados contextos varejistas.

\section{O IMPACTO SOCIAL}

No início da década de 1980, o psicólogo social Bibb Latané (1981) desenvolveu uma teoria do impacto social, a qual especifica o efeito das outras pessoas sobre um indivíduo em particular. O impacto de uma presença social corresponde ao resultado do que o autor chama de "forças sociais", que incluem: tamanho (quantidade de indivíduos); proximidade física (imediatismo) e intensidade das forças sociais (importância que o indivíduo atribui à presença social) (Latané, 1981). A teoria do impacto social estabelece três princípios que definem sua funcionalidade:

- A teoria propõe que o impacto das forças sociais aumenta quando o número de pessoas compartilhando o mesmo espaço é considerado grande, a presença social encontra-se mais próxima fisicamente e é percebida como importante para o indivíduo que a percebe.

- O segundo princípio trata das relações entre as forças sociais. Ele estabelece que o impacto social possui uma função multiplicadora das forças sociais, sugerindo que seu efeito é maior quando há várias pessoas próximas e com alta percepção de importância para o indivíduo. 
- O terceiro e último princípio afirma que a influência da presença social é uma função do número de pessoas, de sua proximidade e importância. Ou seja, o efeito da presença social vai ser difundido ou dividido quando o indivíduo está acompanhado de outras pessoas.

A SIT diz respeito à magnitude do impacto de uma ou mais pessoas (fontes) sobre um indivíduo em particular. As características ligadas à presença de outras pessoas em um ambiente influenciam sentimentos subjetivos individuais, levando a uma série de reações, que incluem, por exemplo, a conformidade e a imitação. Ainda que não ocorra efetiva interação social entre indivíduos que compartilham um dado espaço físico, a presença de outras pessoas ainda é capaz de gerar respostas individuais de cunho emocional, atitudinal e comportamental (Kraut, 1982; Latané \& Nida, 1981; Latané \& Wolf, 1981).

Latané (1981) estabelece que o impacto é uma função multiplicativa de três fatores: $\hat{\imath}=f(S I N)$, onde $\hat{i}$ indica a magnitude do impacto, $f$ denota uma função, o $S$ corresponde às forças das fontes (por exemplo, a sua autoridade ou poder de persuasão), I diz respeito ao imediatismo das fontes (por exemplo, a sua proximidade física ou temporal), e $N$ refere-se ao número de fontes (a quantidade de pessoas, objetivamente).

0 trabalho de Baker (1986) trouxe uma contribuição relevante para o entendimento dos fatores que compõem o ambiente varejista e sua relação com o comportamento do consumidor. Além dos fatores sensoriais e de design, essa pesquisadora destacou a dimensão social como um componente relevante, que, então, passou a ganhar mais atenção por estudiosos da área (exs.: Bitner, 1992; Evans, Christiansen, \& Gill, 1996).

Ressalta-se que, anteriormente à publicação do trabalho de Latané (1981), ainda na década de 1970, o fenômeno denominado crowding foi desenvolvido na psicologia social (Stokols, 1972) e posteriormente adaptado para pesquisas nas áreas de marketing $\mathrm{e}$ comportamento do consumidor. Entretanto, é importante dizer que, neste trabalho, o enfoque não se encontra no impacto do número de consumidores presentes um ambiente de varejo, ainda que essa pista social seja considerada pela SIT. A ênfase encontra-se nas características observáveis de indivíduos (comportamento e aparência em geral) como antecedentes das respostas do consumidor.

Ao passo que os princípios da teoria do impacto social (Latané, 1981) são aplicados em contextos varejistas, contribuições nas áreas de marketing e comportamento do consumidor surgem para melhor compreender as diversas maneiras como uma presença social influencia emoções, atitudes e comportamentos individuais, (exs.: Argo et al., 2005; Argo, Dahl, \& Morales, 2006; Hui, Bradlow, \& Fader, 2009; McFerran \& Argo, 2014), e são esses os aspectos discutidos em mais detalhe a seguir.

\section{PERCEPÇÃO DOS OUTROS CONSUMIDORES NO VAREJO}

A teoria de Latané (1981) tem se tornado referência para estudiosos do comportamento do consumidor interessados em melhor compreender quais fatores sociais interferem nas respostas dos consumidores, bem como de que maneira o fenômeno ocorre. Argo et al. (2005) aplicaram alguns princípios da SIT ao conduzirem um estudo em contexto varejista. Eles verificaram que uma mera presença social (sem alguma interação entre os clientes), em diferentes níveis de tamanho e proximidade, afeta emoções e comportamentos de autoapresentação dos consumidores. A contribuição do trabalho encontra-se no fato de ele apresentar uma investigação inicial sobre o impacto da ausência de interação social em um contexto de consumo, indicando que a simples presença de outros consumidores é capaz de influenciar comportamentos individuais.

Argo et al. (2005) verificaram, ainda, que, diante da presença social (outros clientes), os consumidores se comportaram gerenciando sua impressão. Em situações em que o cliente encontrava-se fisicamente distante das outras pessoas, ou até mesmo sozinho no ambiente, esse tipo apresentou-se menos evidente. 0 gerenciamento de impressões ou autoapresentação consiste em um processo pelo qual as pessoas se comportam de modos específicos para criar uma imagem social desejada perante outras.

Embora consumidores gostem de tocar os produtos durante as compras, Argo et al. (2006) argumentam que o consumidor apresenta avaliações menos favoráveis acerca dos produtos quando percebe que eles foram tocados anteriormente por outros clientes. Contudo, observou-se que, ao perceber que o produto (ex.: peça de vestuário) foi tocado por pessoas julgadas atraentes, as avaliações tendem a ser positivas (Argo, Dahl, \& Morales, 2008).

Juntamente com a proximidade, o tamanho e a importância da presença social, certas características observáveis também são responsáveis por influenciar a maneira como os consumidores avaliam sua experiência de compra (exs.: idade, gênero, comportamento e aparência em geral). Entende-se por pista (estímulo ou elemento) social qualquer característica, comportamento e/ou situação proporcionados por pessoas e/ou grupos, passíveis de interpretação e julgamento, por exemplo: idade, gênero, aparência e interações sociais.

Quando outros clientes são analisados como parte do ambiente, sua influência é manifestada dentro de uma percepção individual sobre eles, baseada em características observadas (Hui et al., 2009; Martin, 1996). Thakor, Suri, e Saleh (2008) verificaram que avaliações acerca do serviço e intenções de compra foram afetadas quando consumidores mais jovens perceberam a presença de consumidores mais velhos (meia-idade e/ou idosos). 
De acordo com Kim e Lee (2012), os outros consumidores correspondem a um construto multidimensional composto pelas seguintes pistas sociais: número de clientes, gênero, comportamento e aparência em geral. Tem-se reconhecido que os outros consumidores impactam avaliações individuais com relação ao varejista, sobretudo quando o cliente identifica (dis)similaridades entre ele e os demais presentes no ambiente (Borges, Chebat, \& Babin, 2010; Söderlund, 2011).

Nesse sentido, a partir da identificação de uma lacuna teórica concernente à ausência de uma escala global utilizada para avaliar as percepções que o indivíduo tem dos outros consumidores durante experiências de consumo, Brocato et al. (2012) estabeleceram três dimensões do conceito que os próprios autores denominaram $\mathrm{OCP}$, e desenvolveram uma escala com o propósito de mensurar esse construto.

Os autores utilizaram a Teoria do Impacto Social (Latané, 1981) como suporte teórico para a definição das pistas sociais antecedentes das dimensões que formam a percepção dos outros consumidores. Os "outros consumidores" são definidos como os consumidores que estão simultaneamente no mesmo ambiente de varejo com o consumidor que percebe sua presença.

De acordo com Brocato et al. (2012), a percepção dos outros consumidores possui três dimensões:

- $\quad$ Similaridade: grau de identificação que o consumidor tem para com os demais consumidores presentes no mesmo ambiente, de se sentir compartilhando a mesma identidade social. Relaciona-se ao quanto o avaliador (consumidores em questão) se sente semelhante e compatível com os demais consumidores.

- Aparência física: características físicas e atributos dos outros clientes percebidos pelo consumidor em questão, por exemplo: idade, gênero e aparência física. Diz respeito aos atributos dos outros consumidores em geral, de modo que as avaliações ao ambiente levam em consideração o quanto o avaliador (consumidor em questão) sente-se bem ao estar com pessoas que ele julga possuírem uma aparência agradável.

- Comportamento adequado: comportamentos ou manifestações compatíveis requeridas dentro de um ambiente social. Em espaços sociais, esperaram-se determinados padrões de comportamento, de modo que ações distintas das almejadas, sobretudo pelo consumidor que avalia sua experiência de consumo também em função dos outros, podem causar desconforto por parte de quem observa. Tal aspecto relaciona-se à existência de normas sociais percebidas e inferidas pelo consumidor.
Cada uma das dimensões foi operacionalmente definida considerando o avaliador (indivíduo que percebe a presença social) e o atributo (a pista social que gera informação ao avaliador). A utilização da escala OCP permite compreender melhor como outros consumidores podem indiretamente influenciar as avaliações pessoais sobre o varejista, sobretudo sua imagem, seus produtos e serviços, além da experiência de compra.

$O$ trabalho de Brocato et al. (2012) amplia o corpo teórico de conhecimento na área, pois, além de consubstanciar trabalhos anteriores que ressaltam a importância da dimensão social do ambiente de varejo (Baker, 1986; Baker, Parasuraman, Grewal, \& Voss, 2002; Grove \& Fisk, 1997; Verhoef et al., 2009), resulta em um instrumento apropriado para entender como os "outros consumidores" influenciam respostas individuais. Em seu estudo, os autores verificaram que comportamentos de aproximação e de afastamento foram influenciados pela maneira como a presença social foi percebida.

Para além das propriedades físicas do ambiente varejista, percepções favoráveis concernentes aos outros consumidores exercem uma forte influência nas respostas afetivas do consumidor (Urich \& Benkenstein, 2012). No contexto varejista, clientes costumam utilizar pistas sociais para formar atitudes e impressões acerca de produtos e serviços (Dahl, Argo, \& Morales, 2012; Žemgulienè, 2013). Raajpoot, Jackson, e Lefebvre (2013) evidenciaram que a similaridade percebida impacta significativamente respostas emocionais, atitudinais e comportamentais.

No contexto brasileiro, Araujo et al. (2013) conduziram a adaptação e a validação da OCP. Esse foi o primeiro estudo no contexto nacional em que a escala foi aplicada. Além de estenderem as possibilidades de verificação do impacto da percepção sobre como os outros clientes influenciam comportamentos individuais de compra (intenções de recompra e de boca a boca positivo), o instrumento foi operacionalizado em um modelo de segunda ordem. As variáveis dependentes investigadas foram significativamente impactadas pelo construto. Argumenta-se que ambientes frequentados por consumidores "similares" conduzem a experiências mais prazerosas, com intenções de comportamento mais favoráveis.

Theodorakis (2014) estendeu o uso da escala OCP ao testar as suas propriedades psicométricas em contexto de centros desportivos. Trezentos e sessenta clientes de três clubes de fitness participaram do estudo. Análise fatorial confirmatória e confiabilidade composta suportaram a validade do construto, e os resultados evidenciaram a plausibilidade da proposição de Brocato et al. (2012). Theodorakis (2014) chama a atenção para o fato de que, apesar de as três dimensões da escala examinarem diferentes elementos relacionados à percepção dos outros consumidores, elas estão fortemente correlacionadas. Logo, a operacionalização do instrumento em um modelo de segunda ordem parece razoável. 


\section{MODELO TEÓRICO E HIPÓTESES DE PESQUISA}

A literatura sobre o comportamento do consumidor em ambientes de consumo sugere que a presença de outros clientes possui influência sobre as emoções e o valor hedônico derivados da compra, pois uma avaliação positiva do indivíduo sobre os outros consumidores (baseada em pistas sociais como idade, gênero, comportamento e aparência em geral) pode deixá-lo mais confortável estando entre eles (Babin, Darden, \& Griffin, 1994; Grove \& Fisk, 1997; Silpakit \& Fisk, 1985; Tombs \& McColl-Kennedy, 2003).

Ao examinarem a influência que as pistas sociais exercem sobre comportamento de compra e imagem de loja, Hu e Jasper (2006) verificaram que atitudes mais favoráveis tendem a ocorrer quando as informações referentes ao ambiente social são bem avaliadas. Argo et al. (2005) também verificaram que uma mera presença social (sem alguma interação entre os clientes) afeta emoções e comportamentos dos consumidores.

Para Söderlund (2011), o comportamento do consumidor é resultante de sua exposição a sutis sinais presentes no ambiente. Logo, os outros clientes são responsáveis por influenciar as respostas individuais. Tombs e McColl-Kennedy (2013) verificaram a existência de uma relação positiva entre avaliações positivas acerca das pistas sociais e respostas emocionais. Brocato et al. (2012) evidenciaram empiricamente que o construto OCP, constituído pelo grau de similaridade percebida entre o consumidor-alvo e os demais consumidores, pela avaliação de suas características físicas e seus comportamentos, relaciona-se com respostas individuais de aproximação e afastamento. Isso implica dizer que avaliações positivas da presença social interferem significativamente na experiência individual de consumo.

Partindo do pressuposto de que os consumidores buscam o prazer até mesmo na compra de itens mais comuns em seu consumo diário, Nuttavuthisit (2014) constatou que os outros consumidores podem contribuir para que experiências estéticas em ambientes varejistas sejam estabelecidas. Por meio de uma série de estudos, McFerran e Argo (2014) demonstraram que a presença de outras pessoas altera sentimentos individuais ao compartilharem o mesmo espaço físico.

Diante do exposto, é razoável assumir que a percepção dos outros consumidores possui similar relacionamento com emoções e valor hedônico resultantes da compra. Portanto, tem-se a Hipótese 1 (relação da OCP com as respostas emocionais) e a Hipótese 2 (relação da OCP com o valor hedônico).

H1: A percepção dos outros consumidores (OCP) possui relação positiva com as respostas emocionais resultantes da compra
H2: A percepção dos outros consumidores (OCP) possui relação positiva com o valor de compra hedônico

As relações propostas, bem como o construto OCP como fator de segunda ordem, estão representadas na Figura 1.

\section{Figura 1. Modelo teórico}

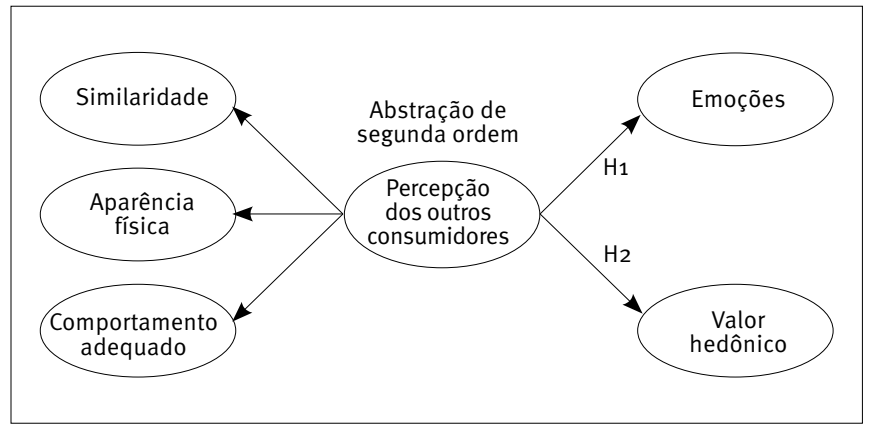

\section{PROCEDIMENTOS METODOLÓGICOS}

Foi realizado um survey do tipo corte transversal e os dados foram coletados por meio de questionários aplicados a uma parte da população-alvo (consumidores de um polo varejista situado no nordeste brasileiro). 0 instrumento de pesquisa foi estruturado em cinco partes, as primeiras três contendo questões referentes à escala OCP (Brocato et al., 2012). Esse construto possui três dimensões:

- $\quad$ Similaridade, contendo cinco perguntas (Sim1: eu pude me identificar com os outros clientes que estavam no centro comercial; Sim2: eu me assemelho a os outros clientes que estavam no centro comercial; Sim3: os outros clientes que estavam no centro comercial são como eu; Sim4: os outros clientes que estavam no centro comercial têm a mesma origem socioeconômica que a minha; Sim5: eu me identifiquei com os outros clientes que estavam no centro comercial).

- $\quad$ Aparência física, com quatro questões (Apf1: eu gostei da aparência física dos outros clientes que estavam no centro comercial; Apf2: os outros clientes estavam vestidos de forma adequada; Apf3: os outros clientes no centro comercial tinham aparência agradável; Apf4: os outros clientes que estavam no centro comercial tinham aparência parecida com a minha).

- Comportamento adequado, também com quatro questões (Comp1: os outros clientes se comportaram adequadamente para um ambiente de compras; Comp2: os outros clientes foram amigáveis comigo; 
Comp3: eu notei que os outros clientes se comportaram bem; Comp4: o comportamento dos outros clientes foi agradável).

A quarta parte contemplou três perguntas relacionadas à dimensão emoções (Richins, 1997) e quatro compreendendo a dimensão valor de compra hedônico (Babin et al., 1994):

- Emoções: (Emo1: alegre; Emo2: animado; Emo3: entusiasmado).

- Valor de compra hedônico: (Vhl1: comprar neste centro comercial foi divertido; Vhl2: eu continuei a fazer compras, não porque tinha que comprar, mas porque senti vontade; Vhl3: comparando com outras coisas que eu poderia ter feito, o tempo passado comprando neste centro comercial foi verdadeiramente agradável; Vhl4: fazer compras neste centro comercial foi uma experiência agradável).

Por último, para fins de caracterização da amostra, cinco perguntas compuseram a quinta parte do instrumento (sexo, escolaridade, renda familiar e idade). Antes da coleta de dados, as escalas foram traduzidas da língua inglesa para o português e, posteriormente, fez-se o inverso, a fim de identificar possíveis discrepâncias no uso de palavras e garantir fidedignidade às perguntas originais. Após o pré-teste do instrumento, não foi verificada a necessidade de alterações no questionário. As escalas foram do tipo Likert de 7 pontos, variando de (1) discordo totalmente a (7) concordo totalmente.

A amostra foi não probabilística e por conveniência, tendo como critério de escolha a acessibilidade e disponibilidade dos sujeitos de pesquisa em colaborar com o estudo. O lócus de investigação foi o Polo Varejista de Caruaru-PE, denominado "o shopping da moda pernambucana". O ambiente possui uma estrutura com $64 \mathrm{mil} \mathrm{m}^{2}$, com mais de 580 lojas, sendo $87 \%$ destas voltadas para vestuário. Pessoas vindas de todo o estado de Pernambuco e também da Paraíba, Alagoas, Ceará, Bahia, Rio Grande do Norte, Piauí, Maranhão, Sergipe, Espírito Santo, Minas Gerais e Pará fazem do centro de compras um dos mais procurados do estado. Os ambientes são cobertos e recebem feiras, exposições, festas e eventos em geral.

A justificativa para a sua escolha encontra-se no fato de os polos varejistas de rua ainda representarem parte considerável do volume de vendas do varejo. Eles continuam sendo uma alternativa para um grande número de empresas varejistas (Parente, Brandão, Miotto, \& Plutarco, 2012). Ademais, há, de maneira geral, uma maior concentração de consumidores compartilhando o mesmo espaço físico, tornando, assim, o contexto adequado para a realização do estudo.
A coleta dos dados ocorreu nos finais de semana dos meses de janeiro e fevereiro de 2014, das 10 às 15 horas, em circunstâncias nas quais o centro comercial encontrava-se com maior fluxo de consumidores. As entrevistas pessoais foram conduzidas sempre após a realização das compras, antes de explicar o propósito do estudo, seu caráter estritamente acadêmico, bem como houve o cuidado quanto à manutenção, em sigilo, da identificação dos entrevistados. Ressalta-se que só foram investigados os consumidores que se inclinaram a colaborar com a pesquisa por livre e espontânea vontade. Os pesquisadores aplicaram os questionários com o auxílio de bolsistas de iniciação científica previamente instruídos para realizar as entrevistas.

Quanto ao tamanho da amostra, inicialmente 300 consumidores a compuseram. Posteriormente, uma inspeção na base de dados revelou ausência de dados, também conhecidos como missing data, além de valores excessivamente reduzidos e elevados, denominados outliers. Quanto ao primeiro caso, optou-se pela imputação de todas as observações com dados perdidos. No que diz respeito aos outliers, os univariados foram identificados a partir das magnitudes de seus escores padronizados, sendo valores de observações superiores a 3 ou inferiores a -3 retirados da amostra (Ribas \& Vieira, 2011).

A estatística útil na identificação de outliers multivariados é a distância de Mahalanobis, a qual mensura cada ponto individual no espaço de $n$ dimensões em relação ao centroide do conjunto de dados (Marôco, 2014). Logo, as observações que apresentaram distâncias significativas $(p<0,001)$ do centroide da amostra foram retiradas (Kline, 2011). Ao final, 247 casos formaram a base de dados.

A análise ocorreu por meio da modelagem de equações estruturais (MEE) baseada em covariâncias, pautada nos princípios de modelagem de segunda ordem e com auxílio de software estatístico. A MEE é uma técnica confirmatória em que o modelo teórico deve ser desenvolvido antes da coleta dos dados, tendo como principal preocupação a confirmação ou não dos dados em termos do padrão de comportamento indicado a partir da argumentação teórica (Brei \& Neto, 2006; Hair, Anderson, Tatham, \& Black, 2005; Hoyle \& Isherwood, 2013). Ela corresponde a uma família de procedimentos relacionados, tendo como função principal a especificação e estimação de modelos que indiquem relações entre variáveis (Kline, 2011).

A modelagem de equações estruturais é cada vez mais usada como um método para a conceituação e desenvolvimento de aspectos teóricos nas ciências sociais aplicadas, em particular na área de marketing. Também, construtos de segunda ordem podem ser modelados fornecendo uma melhor compreensão teórica de relações com boa parcimônia (Hair, Gabriel, \& Patel, 2014, p. 43). 


\section{ANÁLISE E DISCUSSÃO DOS RESULTADOS}

Após a inspeção dos dados (data missing e outliers), as seguintes etapas foram adotadas durante as análises: estatísticas descritivas; análise fatorial exploratória; confiabilidade e validade das escalas usando a consistência interna, confiabilidade composta e variância média extraída; e, finalmente, teste do modelo por meio da modelagem de equações estruturais (Fornell \& Larcker, 1981).

\section{Caracterização da amostra e estatísticas descritivas}

Entre as 247 observações válidas, $64 \%$ são do sexo feminino, o que, de certa maneira, confirma o perfil majoritário de mulheres que frequentam o polo varejista. Considerando que $87 \%$ das lojas são voltadas para o segmento de moda, é comum, sobretudo no interior do nordeste, uma frequência maior da presença feminina no setor varejista. A média de idade dos respondentes é de 31 anos para ambos os grupos.

Quanto à renda, as mulheres possuem média de $R \$$ $1.430,59$, e os homens, $R \$ 1.061,46$. No que diz respeito ao grau de escolaridade dos sujeitos de pesquisa, 56,3\% das mulheres possuem ensino médio completo, $13,9 \%$ cursam graduação e apenas 1,3\% concluiu o nível de pós-graduação. Com relação aos homens, $13,5 \%$ possuem ensino superior completo, $49,4 \%$, ensino médio completo e $25,8 \%$ encontram-se cursando graduação. Com relação à estatística descritiva relacionada aos construtos teóricos utilizados no corrente estudo, a Tabela 1 apresenta três medias de tendência central (média, moda e mediana) e uma de dispersão (desvio padrão).

\section{Tabela 1. Estatísticas descritivas dos construtos}

\begin{tabular}{l|c|c|c|c}
\hline Construto & Média & Moda & Mediana & $\begin{array}{c}\text { Desvio } \\
\text { padrão }\end{array}$ \\
\hline Similaridade & 3,43 & 4 & 3,00 & 0,951 \\
\hline Aparência física & 4,72 & 6 & 5,00 & 1,430 \\
\hline $\begin{array}{l}\text { Comportamento } \\
\text { adequado }\end{array}$ & 4,93 & 6 & 5,00 & 1,209 \\
\hline Emoções & 4,96 & 5 & 5,00 & 0,892 \\
\hline Valor hedônico & 4,80 & 5 & 5,00 & 0,944 \\
\hline
\end{tabular}

De acordo com a Tabela 1, verifica-se uma parcial discordância dos sujeitos entrevistados quanto ao construto similaridade $(3,43)$, ou seja, o grau de identificação do consumidor com os outros consumidores que compartilharam o ambiente físico durante a experiência de compra. Além disso, este foi o construto que obteve o segundo menor desvio padrão. Entende-se que, por se tratar de um espaço com ampla diversidade de lojas e produtos, diferentes públicos o frequentem. Considerando que os polos varejistas são mais democráticos e acessíveis ao público (Parente et al., 2012), parece coerente tal resultado.

Os construtos aparência física $(4,72)$ e comportamento adequado $(4,93)$ obtiveram médias superiores comparativamente à variável similaridade. Analisando os valores da mediana e da moda, pode-se afirmar que, em números absolutos, a maioria dos respondentes julgou positivamente as características físicas e atributos dos outros consumidores (exs.: idade, gênero e aparência física) e seu comportamento. Por entender que há normas sociais subjacentes esperadas em determinados ambientes (Brocato et al., 2012), mesmo se tratando de um ambiente de compras com público consumidor heterogêneo, esses dados parecem indicar uma relativa adequação dos consumidores quanto a comportamentos e indumentárias, características mais facilmente percebidas.

Quanto às respostas emocionais (Média $=4,96$ ) e o valor de compra hedônico (Média $=4,80$ ), os dados da Tabela 1 mostram que essas variáveis seguiram um padrão de concordância parcial (Moda $=5$; Mediana $=5$ ). Assim como os construtos aparência física e comportamento adequado, pelo menos 50\% dos entrevistados responderam positivamente ao contexto de compra.

As emoções desempenham um papel crítico na compreensão do comportamento do consumo, em virtude da forte influência dessa variável nas reações do indivíduo. Nesse mesmo sentido, é coerente inferir que a identificação do consumidor com os outros consumidores pode influenciar as avaliações globais referentes à experiência de consumo, como o valor hedônico atribuído à compra (Babin et al., 1994).

\section{Confiabilidade e validade das escalas}

Para avaliar a adequação dos construtos teóricos, os pesquisadores fizeram uso das análises de validade convergente e discriminante, conforme sugerido por Fornell e Larcker (1981). Antes, objetivando verificar a unidimensionalidade dos construtos, aplicou-se a técnica de análise fatorial exploratória, a qual se propõe a verificar a estrutura de covariâncias entre variáveis em termos de fatores (Malhotra, 2006). A variável 4 da dimensão similaridade foi excluída do modelo por apresentar carga fatorial próxima de o,3 (o que, potencialmente, comprometeria as análises e resultados posteriores).

A partir de então, deu-se início à verificação de validade das escalas. Desse modo, foram conferidas a confiabilidade das escalas (simples e composta) e a variância média extraída. 
Tabela 2. Alpha de Cronbach, Confiabilidade composta e Variância média

\begin{tabular}{l|c|c|c|c}
\hline Construto & $\begin{array}{c}\text { № de } \\
\text { ítens }\end{array}$ & Alfa & CC & AVE \\
\hline Similaridade & 4 & 0,85 & 0,847 & 0,52 \\
\hline Aparência física & 4 & 0,94 & 0,867 & 0,65 \\
\hline $\begin{array}{l}\text { Comportamento } \\
\text { adequado }\end{array}$ & 4 & 0,95 &, 9370 & 0,64 \\
\hline Emoções & 3 & 0,85 & 0,858 & 0,61 \\
\hline Valor hedônico & 4 & 0,82 & 0,733 & 0,54 \\
\hline
\end{tabular}

Notas: Alfa = Alpha de Cronbach; $C C=$ Confiabilidade Composta; $A V E=$ Variância Média Extraída.

É possível verificar, a partir da Tabela 2, que todos os valores do Alpha de Cronbach obtiveram resultados satisfatórios. Essa medida varia entre o e 1, sendo que, acima de 0,70, considera-se a confiabilidade da escala (Hair et al., 2005). Entretanto, este não foi o único critério adotado no exame das escalas no modelo.
Examinou-se a confiabilidade composta objetivando analisar os construtos de maneira mais cuidadosa.

Os indicadores também se mostraram adequados, especialmente quando se observa a variância média extraída (AVE), que corresponde a uma medida indicativa de confiabilidade do modelo dos construtos. O valor mínimo considerado para a AVE é 0,50 (Hair et al., 2005; Kline, 2011). Conforme pode ser observado na Tabela 2, todas as variáveis latentes obtiveram o mínimo aceitável, de modo que a validade convergente foi atendida.

A validade discriminante foi realizada comparando-se a variância compartilhada entre cada par de construto com a AVE de cada construto do par, seguindo o procedimento proposto por Fornell e Larcker (1981). Espera-se que a medida em questão (AVE) não apresente valores de correlação altos com outras variáveis das quais teoricamente difere. A validade discriminante objetiva verificar em que medida as escalas medem o que se propõem medir, ou seja, espera-se que nessa análise os construtos não se correlacionem fortemente entre si.

Tabela 3. Correlações, Variância compartilhada e AVE

\begin{tabular}{l|c|c|c|c|c}
\hline Variáveis & Similaridade & Aparência física & Comportamento adequado & Emoções & Valor hedônico \\
\hline Similaridade & 0,52 & $0,53^{\star \star}$ & $0,46^{\star \star}$ & $0,35^{\star \star}$ & $0,32^{\star \star}$ \\
\hline Aparência física & 0,28 & 0,65 & $0,83^{\star \star}$ & $0,53^{\star \star}$ & $0,38^{\star \star}$ \\
\hline Comportamento adequado & 0,21 & 0,68 & 0,64 & $0,54^{\star \star}$ & $0,45^{\star *}$ \\
\hline Emoções & 0,12 & 0,28 & 0,29 & 0,61 & $0,65^{\star \star}$ \\
\hline Valor hedônico & 0,10 & 0,14 & 0,20 & 0,42 & 0,54 \\
\hline
\end{tabular}

Nota: os valores na diagonal mostram a AVE de cada construto, os valores acima da diagonal mostram as correlações entre os construtos e os valores abaixo da diagonal, a variância compartilhada (correlações ao quadrado).

Observa-se pela Tabela 3 que os valores da variância média extraída são maiores que a variância compartilhada em cada par de constructo. A única exceção foi o valor da correlação entre as dimensões comportamento adequado e aparência física. Quando testou as propriedades psicométricas da escala OCP, Theodorakis (2014) chamou a atenção para o fato de que, apesar de as três dimensões da escala examinarem diferentes elementos relacionados à percepção dos outros consumidores, elas estão fortemente correlacionadas.

Desse modo, entende-se que valores altos da correlação entre as dimensões da OCP possam ser encontrados, maiores até que a própria variância compartilhada dos pares de cada fator. Farrell (2013) evidencia problemas no uso da comparação entre AVE e variância compartilhada como critério para aferir a validade discriminante. Segundo esse autor, variáveis observadas podem assumir indicadores baixos, porém consistentes com seus respectivos construtos, mas, quando os construtos distintos são correlacionados, índices elevados podem surgir.

Entende-se que a operacionalização das dimensões da OCP em um modelo de segunda ordem mitiga o aparente problema encontrado nos parâmetros da correlação entre os fatores comportamento adequado e aparência física, uma vez que, em última instância, as três dimensões formam um único construto (percepção dos outros consumidores). Portanto, é razoável assumir que a validade discriminante também foi atendida. Em face disso, a próxima etapa, então, foi verificar o modelo proposto.

\section{Análise do modelo estrutural}

Após verificação da validade convergente e discriminante, procedeu-se ao teste do modelo estrutural (Anderson \& Gerbing, 1988; 
Bagozzi \& Yi, 2012). A técnica de estimação por máxima verossimilhança, amplamente utilizada nos estudos em marketing e comportamento do consumidor (exs.: Almeida, Mazzon, Neto, \& Dholakia, 2012; Byun \& Mann, 2011; Vieira, 2009), foi escoIhida para verificação das estimativas e índices de ajustamento. 0 modelo estrutural estimado é apresentado por meio dos índices de ajuste na Tabela 4.

Tabela 4. Índices de ajustamento do modelo

\begin{tabular}{c|c|c}
\hline Índices & Critério & Modelo estrutural \\
\hline $\mathrm{X}^{2}$ & -- & 303,077 \\
\hline $\mathrm{Gl}$ & -- & 141 \\
\hline $\mathrm{p}$-value & $>0,05$ & 0,001 \\
\hline $\mathrm{X}^{2} /(\mathrm{gl})$ & $\leq 3$ & 2,149 \\
\hline $\mathrm{NFI}$ & $\geq 0,900$ & 0,933 \\
\hline $\mathrm{IFI}$ & $\geq 0,900$ & 0,963 \\
\hline $\mathrm{TLI}$ & $\geq 0,900$ & 0,955 \\
\hline $\mathrm{CFI}$ & $\geq 0,900$ & 0,963 \\
\hline $\mathrm{RMSEA}$ & $\leq 0,08$ & 0,068 \\
\hline
\end{tabular}

Conforme pode ser observado, o modelo obteve qui-quadrado $\left(\mathrm{x}^{2}\right)$ igual a 303,077 e 141 graus de liberdade (gl). A consistência global do modelo é verificada inicialmente pelo valor do $x^{2}$. Em princípio, a sua magnitude $(p=0,001)$ indica um ajuste inadequado. Entretanto, vale ressaltar que faz-se necessária cautela na interpretação do $p$-value, pois essa medida é bastante influenciada pelo tamanho da amostra (Vieira, Pires, \& Galeano, 2013). Quando o tamanho da amostra é suficientemente grande ( $>$ > 200), o que constitui uma condição necessária para aferir robustez ao teste estatístico, com frequência, o $p$-value é significativo.

Objetivando reduzir a sensibilidade da estatística $x^{2}$ ao tamanho da amostra, estudiosos propõem considerar a razão do valor do qui-quadrado pelos graus de liberdade. Embora não exista uma regra geral que aponte o valor mínimo aceitável para $\mathrm{x}^{2} /(\mathrm{gl})$, o critério comumente empregado é que essa razão seja menor ou igual do que 3 (Kline, 2011). Nesse sentido, é possível considerar essa medida satisfatória $\left(\mathrm{x}^{2} / \mathrm{gl}=2,149\right)$.

Quanto aos índices de ajustamento (NFI, IFI, TLI e CFI) que comparam o modelo proposto com o modelo nulo, verificou-se que todos apresentaram valores de adequação superiores aos sugeridos pela literatura. No que diz respeito ao RMSEA (raiz do erro quadrático médio de aproximação), entende-se que este também apresenta ajustamento aceitável. Índices cujos valores sejam inferiores a 0,05 indicam um bom ajustamento. Valores entre 0,05 e 0,08 são considerados aceitáveis e valores variando entre 0,08 e 0,10 sugerem ajustes pobres (Arbuckle, 2012). Logo, os resultados indicam um ajuste satisfatório. As hipóteses do modelo original proposto foram testadas a partir da avaliação da significância dos parâmetros padronizados (Tabela 5).

Tabela 5. Teste das hipóteses

\begin{tabular}{|c|c|c|c|c|c|}
\hline $\mathrm{H}_{1} \mathrm{OCP} \ldots . . \mathrm{Emocões}$ & 0,76 & 0,055 & 0,77 & 13,216 & 0,001 \\
\hline H2 OCP .... Valor hedônico & 0,57 & 0,065 & 0,56 & 8,842 & 0,001 \\
\hline
\end{tabular}

Verificou-se que as duas hipóteses se confirmaram, dado que os valores do teste $t$ encontram-se dentro dos intervalos propostos pela literatura $t>1,96$ e $p \leq 0,05$ (Hair et al., 2005). Isso significa dizer que a percepção do consumidor com relação aos outros consumidores presentes no mesmo ambiente varejista, a partir de similaridades, características físicas e comportamento observados, possui relação positiva com as respostas emocionais positivas e o valor de compra hedônico. Ademais, as emoções $\left(R^{2}=0,37\right)$ e o valor hedônico $\left(R^{2}=0,29\right)$ são explicados pela percepção dos outros consumidores (Figura 2).

Figura 2. Modelo estrutural final

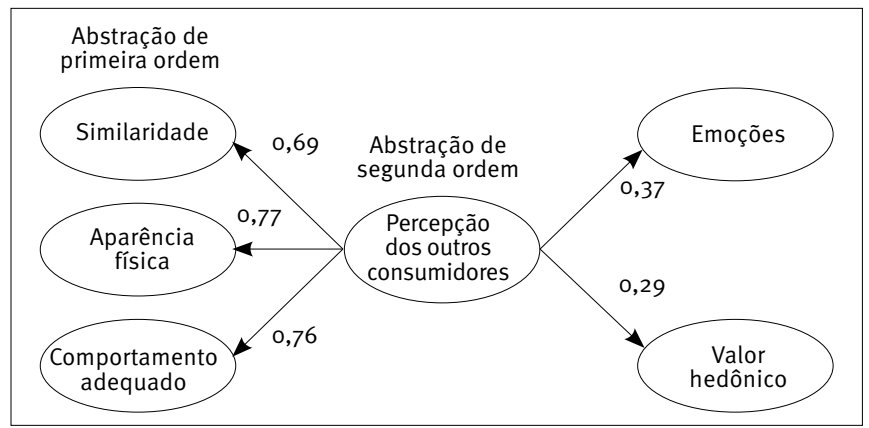


Ainda no que diz respeito ao modelo, evidenciou-se que as dimensões similaridade, aparência física e comportamento adequado compõem adequadamente o construto OCP, ao passo que se tornam variáveis dependentes, sendo a variação explicada pelo fator de ordem superior, ou seja, o construto contendo suas três dimensões. Assim, o estudo evidencia o potencial que a percepção da presença de "outros consumidores" possui no tocante a melhorar ou diminuir as avaliações individuais acerca da experiência de consumo e o ambiente varejista em si (Argo et al., 2005; Brocato et al., 2012).

Características dos outros consumidores, tais como idade, gênero, comportamento e aparência, em geral são frequentemente utilizadas para auxiliar o consumidor a inferir a imagem da loja (Dahl et al., 2012; Žemgulienè, 2013). Além disso, percepções favoráveis concernentes aos outros consumidores exercem uma forte influência nas respostas afetivas do consumidor (Uhrich \& Benkenstein, 2012). Apesar de não ter sido verificado o efeito direto da dimensão similaridade sobre as respostas emocionais e o valor de compra hedônico, os resultados sugerem um alinhamento aos achados do estudo de Raajpoot et al. (2013), o qual verificou impacto significativo da similaridade percebida dos consumidores sobre respostas individuais de cunho emocional, atitudinal e comportamental.

Os resultados ainda vão ao encontro das preocupações de autores como Grove e Fisk (1997), Baker et al. (2002) e Veorhef et al. (2009), no que se diz respeito à importância que deve ser atribuída à percepção dos outros consumidores para entender as variações nas avaliações feitas pelo consumidor sobre sua experiência de compra em ambiente varejista.

\section{CONCLUSÕES}

O presente estudo investigou a relação da percepção da presença de outros clientes com as emoções e o valor hedônico, resultantes da compra em ambiente de varejo popular. Os resultados evidenciam índices significativos para as dimensões do construto teórico, similares aos encontrados no estudo de Araujo et al. (2013). A aplicação em um polo varejista com diversidade em termos de perfil de clientes e tipos de loja mostrou-se adequada.

0 estudo traz uma contribuição relevante para o campo de estudos do comportamento do consumidor em ambientes de varejo, pois a aplicação e validação da escala em varejo tradicional amplia as possibilidades de investigar o impacto que a dimensão social assume nos mais variados contextos varejistas. Assim como na aplicação original da escala, o estudo consubstancia o argumento de que a experiência de consumo envolve mais que uma simples avaliação do desempenho do varejista.
Além de fornecer novas evidências do impacto da OCP sobre o comportamento individual de consumo, o estudo também verifica o efeito de respostas do consumidor que, até o momento, não tinham sido avaliadas (emoções e valor de compra hedônico). A aceitação da presença de outros clientes possui impacto nas emoções e pode gerar atitudes mais favoráveis quando o ambiente social é bem-avaliado (Hu \& Jasper, 2006; Tombs \& McColl-Kennedy, 2003).

Assim como na aplicação original da escala (Brocato et al., 2012), este trabalho valida empiricamente algumas ideias presentes no modelo de Verhoef et al. (2009), sobretudo aquela que apreende a experiência de consumo como mais que apenas uma simples avaliação do desempenho do varejista. Fatores como renda, gênero, classe social e traços de personalidade possam interferir na maneira como a presença social é percebida e avaliada.

Acredita-se que o varejo brasileiro, como mercado emergente, passa por um momento de transformações, sobretudo por conta da sua heterogeneidade (Seth, 2011) e do crescimento da renda dos consumidores da base da pirâmide (Parente et al., 2012). Consequentemente, diferentes perfis de consumidores, nos mais diversos aspectos, estão compartilhando os mesmos ambientes varejistas. $O$ fato de a presença de outros consumidores ter impacto tão forte em comportamentos futuros sugere a necessidade de uma atenção especial a esse tema (Araujo et al., 2013).

Ressalta-se que, pela limitação do estudo, não é possível generalizar os resultados encontrados, tendo em vista que a amostra utilizada foi do tipo não probabilística por acessibilidade. Apesar de os índices de ajustamento do modelo atenderem aos requisitos para a $M E E$, recomenda-se considerar os resultados no contexto específico em que a escala foi aplicada.

Espera-se que o trabalho possa estimular a realização de investigações posteriores a fim de verificar antecedentes e demais consequentes relacionados ao construto OCP, bem como a aplicação da escala em ambientes varejistas diversos, objetivando encontrar evidências que confirmem ou refutem sua utilização em contextos específicos.

\section{Nota da Redação}

Este artigo foi apresentado no $7^{\underline{0}}$ Congresso LatinoAmericano de Varejo: Varejo e Desenvolvimento em Mercados Emergentes (CLAV) em 2014, organizado pelos professores Delane Botelho e Edgard Barki, promovido pelo Centro de Excelência em Varejo da FGV/EAESP (GVcev) 


\section{REFERÊNCIAS}

Aguiar, E. C., \& Gomes, V. M. S. (2014). Percepção de crowding e comportamento do consumidor: Uma abordagem não linear no varejo supermercadista de baixa renda. Anais do 6을 Encontro de Marketing da ANPAD, Gramado, RS.

Almeida, S. O., Mazzon, J. A., Neto, H. F. M., \& Dholakia, U. (2012). Efeitos da comunidade de origem no participante de comunidades virtuais de marca. RAE-Revista de Administração de Empresas, 52 (2), 204-216. doi:10.1590_0034-7590201200020000

Anderson, J. C., \& Gerbing, D. W. (1988). Structural equation modeling in practice: A review and recommended two-step approach. Psychological Bulletin, 103(3), 411-423. doi:10.1037/0033-2909.103.3.411

Araujo, C. F., Grillo, T. L. H., \& Espartel, L. B. (2013). Influência da percepção de outros clientes nas intenções de comportamento do consumidor: Validação da escala OCP no contexto brasileiro e teste de um modelo de segunda ordem. Anais do 370 Encontro da ANPAD, Rio de Janeiro.

Arbuckle, J. L. (2012). AMOSTM 21 user's guide (21a ed.). Chicago: SPSS.

Argo, J. J., Dahl, D. W., \& Manchanda, R. V. (2005). The influence of a mere social presence in a retail context. Journal of Consumer Research, 32(2), 207-212. doi:10.1086/432230

Argo, J. J., Dahl, D. W., \& Morales, A. C. (2006). Consumer contamination: How consumers react to products touched by others. Journal of Marketing, 70(2), 81-94. doi:10.1509/jmkg.70.2.81

Argo, J. J., Dahl, D. W., \& Morales, A. C. (2008). Positive consumer contagion: Responses to attractive others in a retail context. Journal of Marketing Research, 45(6), 690-701. doi:10.1509/jmkr.45.6.690

Babin, B. J., Darden, W. R., \& Griffin, M. (1994). Work and/or fun: Measuring hedonic and utilitarian shopping value. The Journal of Consumer Research, 20(4), 644-656.

Bagozzi, R. P., \& Yi, Y. (2012). Specification, evaluation, and interpretation of structural equation models. Journal of the Academy of Marketing Science, 40(1), 8-34. doi:10.1007/s11747-011-0278-x

Baker, J. A. (1986). The role of the environment in marketing services: The consumer perspective. In The Services Challenge: Integrating for Competitive Advantage. Eds John A. Czepeil, Carole A. Congram, \& James Sha/lahan. Chicago: American Marketing Association, 79-84.

Baker, J. A., Parasuraman, A., Grewal, D., \& Voss, G. (2002). The influence of multiple store environment cues on perceived merchandise value and patronage intentions. Journal of Marketing, 66(2), 120-141. doi:10.1509/jmkg.66.2.120.18470

Bitner, M. J. (1990). Evaluating services encounters: The effects of phys ical surroundings and employee responses. Journal of Marketing, $54(2), 69-82$

Bitner, M. J. (1992). Servicescapes: The impact of physical surroundings on customers and employees. Journal of Marketing, 56(2), 57-71. doi:10.2307/1252042

Borges, A., Chebat, J., \& Babin, B. J. (2010). Does a companion always enhance the shopping experience? Journal of Retailing and Consumer Services, 17(4), 294-299. doi:10.1016/j.jretconser.2010.02.007

Brandão, M. M., \& Parente, J. (2012). Brasileiro gosta de "muvuca"? Impacto da densidade humana no comportamento de compra. RAE-Revista de Administração de Empresas, 52(6), 613-627. doi:10.1590_0034-7590201200600004
Breazeale, M., \& Ponder, N. (2013). Get the picture? Visual servicescapes and self-image congruity. Journal of Business Research, 66(7), 839-846. doi:10.1016/j.jbusres.2011.06.009

Brei, V. A., \& Neto, G. L. (2006). O uso da técnica de modelagem em equações estruturais na área de marketing: Um estudo comparativo entre publicações no Brasil e no exterior. RAC-Revista de Administração Contemporânea, 10(4), 131-151. doi:10.1590/s141565552006000400007

Brocato, E. D., Voorhees, C. M., \& Baker, J. (2012). Understanding the influence of cues from other customers in the service experience: $A$ scale development and validation. Journal of Retailing, 88(3), 384398. doi:10.1016/j.jretai.2012.01.006

Byun, S., \& Mann, M. (2011). The influence of others: The impact of perceived human crowding on perceived competition, emotions, and hedonic shopping value. Clothing and Textiles Research Journal, 29(4), 284-297. doi:10.1177/0887302X11422820

Dahl, D. W., Argo, J. J., \& Morales, A. C. (2012). Social information in the retail environment: The importance of consumption alignment, referent identity, and self-esteem. Journal of Consumer Research, 38(5), 860-871. doi:10.1086/660918

Eroglu, S. A., Machleit, K. G. D., \& Barr, T. F. (2005). Perceived retail crowding and shopping satisfaction: The role of shopping calues. Journal of Business Research, 58(8), 1146-1153. doi:10.1016/j.jbusres.2004.01.005

Evans, K. R., Christiansen, T., \& Gill, J. D. (1996). The impact of social influence and role expectations on shopping center patronage intentions. Journal of the Academy of Marketing Science, 24(3), 208-218. doi:10.1177/0092070396243002

Farrell, A. M. (2013). Insufficient discriminant validity: A comment on Bove, Pervan, and Shiu (2009). Journal of Business Research, 63(1), 324-327. doi:10.1016/j.jbusres.2009.05.003

Fornell, C., \& Larcker, D. F. (1981). Evaluating structural equation models with unobservable variables and measurement error. Journal of Marketing Research, 18(1), 39-50. doi:10.2307/3151312

Grove, S. J., \& Fisk, R. P. (1997). The impact of other customers on service experiences: A critical incident examination of "getting along". Journal of Retailing, 73(1), 63-85. doi:10.1016/s0022-4359(97)90015-4

Hair, J. F., Anderson, R. E., Tatham, R. L., \& Black, W. C. (2005). Análise multivariada de dados (4th ed.). Englewood Cliffs-NJ: Prentice-Hall.

Hair, J. F., Gabriel, M. L. D. S., \& Patel, V. K. (2014). Modelagem de equações estruturais baseada em covariância (CB-SEM) com o AMOS: Orientações sobre a sua aplicação como uma ferramenta de pesquisa de marketing. REMark-Revista Brasileira de Marketing, 13(2), 43-53. doi:10.5585/remark.v13i2.2718

Hoyle, R. H., \& Isherwood, J. C. (2013). Reporting results from structural equation modeling analyses in archives of scientific psychology. American Psychological Association, 1(1), 14-22. doi:10.1037/ arcoooooo4

Hu, H., \& Jasper, C. R. (2006). Social cues in the store environment and their impact on store image. International Journal of Retail \& Distribution Management, 34(1), 25-48. doi:10.1108/09590550610642800

Hui, S. K., Bradlow, E. T., \& Fader, P. S. (2009). Testing behavioral hypotheses using an integrated model of grocery store shopping path and purchase behavior. Journal of Consumer Research, 36(3), 478493. doi:10.1086/599046

Kim, J., \& Kim, J. (2012). Human factors in retail environments: A review. International Journal of Retail \& Distribution Management, 40(11), 818-841. doi:10.1108/09590551211267593 
Kim, N., \& Lee, M. (2012). Other customers in a service encounter: Examining the effect in a restaurant setting. Journal of Service Marketing, 26(1), 27-40. doi:10.1108/08876041211199706

Kline, R. B. (2011). Principles and practice of structural equation modeling (3rd ed.). New York-NY: The Guilford Press.

Kraut, R, E. (1982). Social presence, facial feedback, and emotion. Journal of Personality and Social Psychology, 42(5), 853-863. doi:10.1037/0022-3514.42.5.853

Latané, B. (1981). The psychology of social impact. American Psychologist, 36(4), 343-356. doi:10.1037/0003-066x.36.4.343

Latané, B., \& Nida, S. (1981). Ten years of research on group size and helping. Psychological Bulletin, 89(2), 308-324. doi:10.1037/00332909.89.2.308

Latané, B., \& Wolf, S. (1981). The social impact of majorities and minorities. Psychological Review, 88(5), 438-453. doi:10.1037/0033$295 \times .88 .5 .438$

Malhotra, N. K. (2006). Pesquisa de marketing: Uma orientação aplicada (4a ed.). São Paulo: Prentice Hall.

Marôco, J. (2014). Análises de equações estruturais: Fundamentos teóricos, software e aplicações (2a ed.). Pêro Pinheiro: Report Number.

Martin, C. L. (1996). Consumer-to-consumer relationships: Satisfaction with other consumers' public behavior. Journal of Consumer Affairs, 30(1), 146-169. doi:10.1111/j.1745-6606.1996.tboo729.x

McFerran, B., \& Argo, J. J. (2014). The entourage effect. Journal of Consumer Research, 40(5), 871-884. doi:10.1086/673262

McGrath, M. A., \& Otnes, C. (1995). Unacquainted influencers: When strangers interact in retail setting. Journal of Business Research, 32(3), 261-272. doi:10.1016/0148-2963(94)00051-f

Nuttavuthisit, K. (2014). How consumers as aesthetic subjects cocreate the aesthetic experience of the retail environment. Journal of Retailing and Consumers Services, 21(4), 432-437. doi:10.1016/j. jretconser.2014.03.003

Parente, J., Brandão, M. M., Miotto, A. P., \& Plutarco, F. (2012). Main street retail districts or shopping centers? comparing the preferences of low-income consumers. BBR-Brazilian Business Review, 9(1), 154179. doi:10.15728/bbrconf.2012.7

Raajpoot, N., Jackson, A., \& Lefebvre, J. (2013). Non-verbal customer-to-customer interaction in retail setting: An investigation of indirect effects of perceived customer similarity on important marketing outcomes. Atlantic Marketing Journal, 2(1), 16-41.

Ribas, J. R., \& Vieira, P. R. C. (2011). Análise multivariada com o uso do SPSS. Rio de Janeiro: Editora Ciência Moderna.

Richins, M. L. (1997). Measuring emotions in the consumption experience. Journal of Consumer Research, 24(2), 127-146. doi:10.1086/209499

Sheth, J. N. (2011). Impact of emerging markets on marketing: Rethinking existing perspectives and practices. Journal of Marketing, 75(4), 166-182. doi:10.1509/jmkg.75.4.166

Silpakit, P., \& Fisk, R. P. (1985). Participatizing the service encounter: A theoretical framework. In T. M. Block, G. D. Upah, \& V. A. Zeithaml (Org.). Services marketing in a changing environment (pp. 117-121). Chicago, IL: American Marketing Association.
Söderlund, M. (2011). Other customers in the retail environment and their impact on the customer's evaluations of the retailer. Journal of Retailing and Consumer Services, 18(3), 174-182. doi:10.1016/j.jretconser.2010.09.006

Stokols, D. (1972). On the distinction between density and crowding: Some implications for future research. Psychological Review, 79(3), 275-277. doi:10.1037/hoo32706

Thakor, M. V., Suri, R., \& Saleh, K. (2008). Effects of service setting and other consumers' age on the service perceptions of young consumers. Journal of Retailing, 84(2), 137-149. doi:10.1016/j.jretai.2008.01.002

Theodorakis, N. D. A (2014). A cross-validation study of the other customers perceptions scale in the context of sport and fitness centres. Revista Internacional de Ciencias del Desporte, 10(35), 63-74.

Tombs, A. G., \& McColl-Kennedy, J. R. (2003). Social-servicescape: Conceptual model. Marketing Theory, 3(4), 447-475. doi:10.1177/1470593103040785

Tombs, A. G., \& McColl-Kennedy, J. R. (2010). Social and spatial influence of customers on other customers in the social-servicescape. Australasian Marketing Journal, 18(3), 120-131. doi:10.1016/j.ausmj.2010.04.001

Tombs, A. G., \& McColl-Kennedy, J. R. (2013). Third party customers infecting other customers for better or for worse. Psychology and Marketing, 30(3), 277-292. doi:10.1002/mar.20604

Turley, L. W., \& Milliman, R. E. (2000). Atmospheric effects on shopping behavior: A review of the experimental evidence. Journal of Business Research, 49(2), 193-211. doi:10.1016/S0148-2963(99)00010-7

Uhrich, S., \& Benkenstein, M. (2012). Physical and social atmospheric effects in hedonic service consumption: Customers' roles at sporting events. The Service Industries Journal, 32(11), 1741-1757. doi:10.1080 /02642069.2011.556190

Verhoef, P. C., Lemon, K. N., Parasuraman, A., Roggeveen, A., Tsiros, M., \& Schlesinger, L. A. (2009). Customer experience creation: Determinants, dynamics and management strategies. Journal of Retailing, 85(1), 31-41. doi:10.1016/j.jretai.2008.11.001

Vieira, V. A. (2009). Moderação, mediação, moderadora-mediadora e efeitos indiretos em modelagem de equações estruturais: Uma aplicação no modelo de desconfirmação de expectativas. RAUSP-Revista de Administração da Universidade de São Paulo, 44(1), 17-33.

Vieira, V. A., Pires, D., \& Galeano, R. (2013). Determinantes do desempenho empresarial e das vendas cruzadas no varejo. RAE-Revista de Administração de Empresas, 53(6), 565-579. doi:10.159o/so034759020130605

Yüksel, A. (2009). Exterior color and perceived retail crowding: Effects on tourists' shopping quality inferences and approach behaviors. Journal of Quality Assurance in Hospitality \& Tourism, 10(4), 233-254. doi:10.1080/15280080903183383

Žemgulienè, J. (2013). Relative importance of retail store image and consumers characteristics on the perception of value and willingness to pay a premium price. Regional Formation and Development Studies, 9(1), 157-165. doi:10.15181/rfds.vgi1.602 